\title{
On the 1994 Outbursts of GRO J1655-40
}

\author{
Kinwah Wu and Richard W. Hunstead
}

School of Physics, University of Sydney, NSW 2006, Australia

\begin{abstract}
.
We discuss some of the issues raised by the X-ray, radio and optical observations made during the 1994 outbursts of GRO J1655-40.
\end{abstract}

\section{Introduction}

The candidate black hole binary GRO J1655-40 was discovered as an X-ray transient by BATSE on 1994 July 27 (Harmon et al. 1995). Three X-ray outbursts were recorded by BATSE in the 150 days after its discovery, with the third being the strongest. Corresponding radio outbursts were detected by the Molonglo Observatory Synthesis Telescope (MOST) at $843 \mathrm{MHz}$ (Hunstead et al. 1995) and by the VLA at higher frequencies (Hjellming and Rupen 1995).

\section{Observations}

Following its discovery as a strong radio source, GRO J1655-40 was monitored closely by the MOST at $843 \mathrm{MHz}$; the MOST and BATSE light curves are shown in Figure 1. Closer examination shows that each major radio event recorded by MOST is composed of two separate components, one with a decay time of $\sim 4.5$ days per decade of flux density and the other $\sim 20$ days. The VLA sampling intervals were too coarse to reveal this shorter timescale component for the first two outbursts. A MOST-VLA comparison for the third outburst, however, suggests that the $843 \mathrm{MHz}$ behaviour is different from that seen at frequencies $>1 \mathrm{GHz}$.

During the period 1994 Aug 26-Sep 6, GRO J1655-40 was observed with the Anglo-Australian Telescope in two spectral regions, one centred at $\mathrm{H} \alpha$ and the other at $\mathrm{HeII} / \mathrm{H} \beta$. At this time the source was in the decay phase from its first radio outburst and the BATSE signal was low. The Aug 30-Sep 4 co-added $\mathrm{HeII} / \mathrm{H} \beta$ spectrum was rich in high excitation lines, strongly resembling that of a WN Wolf-Rayet star (see Hunstead, this volume). On September 6, at the onset of the second X-ray outburst, the Wolf-Rayet features disappeared, leaving the spectrum dominated by lower excitation Balmer lines.

\section{Discussion}

While the longer timescale component of the radio outbursts is most likely due to synchrotron emission from the relativistic jets, the origin of the shorter timescale 


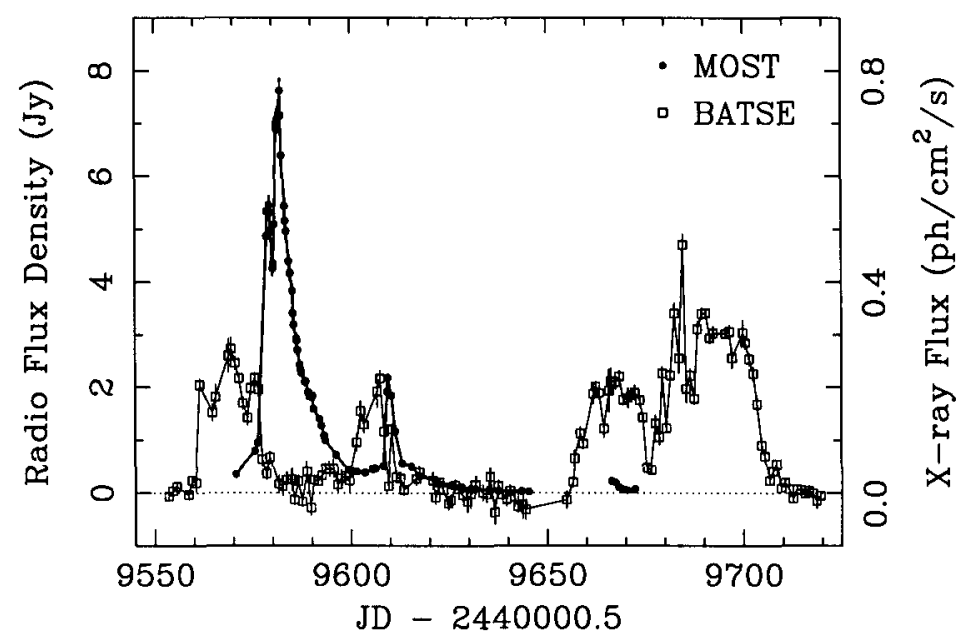

Figure 1. The $843 \mathrm{MHz}$ radio and $20-100 \mathrm{keV} \mathrm{X}$-ray light curves for GRO J1655-40 during the 1994 outbursts.

component remains uncertain. The radio spectrum suggests either sychrotron emission from the injection of a short-lived population of low-energy electrons or possibly free-free emission from a hot corona. The Wolf-Rayet features in the optical spectrum obtained before the onset of the second X-ray outburst are indicative of a hot dense outflow from the accretion disk surrounding the black hole, implying the presence of a hot disk corona. The sudden disappearance of these features could be explained by disruption of the corona associated with the X-ray turn-on.

Although the timing of the radio outbursts seems to correlate with and lag the X-ray outbursts, there is no obvious correlation between their strengths. In fact, while the radio intensities decreased monotonically, the third X-ray outburst was more energetic than the sum of the two preceding outbursts. This behaviour could be explained by depletion of the store of energetic electrons in the disk corona as a consequence of the X-ray outbursts.

We speculate that if the first two X-ray oubursts were caused by the same mechanism - instability of the accretion disk surrounding the black hole - the $\mathrm{X}$-ray flux would be sufficient to irradiatively heat the secondary star, causing an enhancement in the mass transfer and inducing an even stronger third outburst.

We thank the BATSE team for providing us with the data shown in Fig 1.

\section{References}

Harmon, B. A. et al. 1995, Nature, 374, 303

Hjellming, R. M. and Rupen, M. P. 1995, Nature, 375, 8

Hunstead, R. W. et al. 1995, Compact Stars in Binaries, eds. J. van Paradijs et al., Dordrecht: Kluwer, p.369 